\title{
Research on Judicial Practice of School Physical Education Injury Cases in Jiangxi Province—Statistical Analysis Based on the Big Data of China Judgement Online
}

\author{
Jianxin $\mathrm{Gao}^{1, \mathrm{a} *}$, Yuan $\mathrm{Shu}^{2}$, Liya $\mathrm{Li}^{3}$, Zhuo Huang ${ }^{4}$ \\ ${ }^{1}$ Department of sports, Jiangxi Teachers College, Yingtan, Jiangxi, China \\ ${ }^{2}$ Department of sports, Jiangxi Teachers College, Yingtan, Jiangxi, China \\ ${ }^{3}$ Department of sports, Jiangxi Teachers College, Yingtan, Jiangxi, China \\ ${ }^{4}$ Institute of Physical Education, Nanchang University, Nanchang, Jiangxi, China
}

\begin{abstract}
Justice is the last line to maintain social fairness and justice". Student right for life, body and health can only be guaranteed through judicial channels. Once the school sports injury cases happen, the results are serious, which seriously affects the students' academic completion, and brings heavy economics and psychological burden to the related families. Therefore, from the perspective of big data analysis, this paper collects and sorts out the data reports of China judgement online, and finds problems from the data statistics of school sports injury cases. By using SPSS and other sports statistics related software to analyze the variables involved in the case, the paper truthfully reflects the current situation and problems of the case. Finally, the paper puts forward countermeasures and suggestions to prevent and solve the school sports injuries in Jiangxi Province, in order to provide theoretical reference for the construction of school sports law in China.
\end{abstract}

\section{Introduction}

In recent years, under the guidance of the concept of "health first", school physical education in China has made rapid development. With the high attention of the national education department and the sports department, some school sports policies and regulations have been implemented, the number and time of students participating in physical exercises at all levels are increasing, and the awareness of students participating in physical exercises is also increasing. However, due to the particularity of sports, sports injury cases occur frequently in school sports [1]. According to statistics, nearly 100 cases occur in all kinds of schools in China every year more than 50 cases of school sports injuries in Jiangxi province in recent years. The occurrence of school sports injury cases has brought serious impact on students, families, schools and society. The reason is due to the inadequate supervision and education of school sports and the lack of students' awareness of sports safety. The main reason is that the legal and regulatory system of school sports in China is not perfect [2].

\section{Research object and method}

The time retrieval scope of the case is limited to the school sports injury cases heard by the people's courts at all levels of Jiangxi province from 2015 to 2019. The research object of this paper is based on the national public judgment documents to ensure the authenticity and reliability of the research cases. The main research methods used in this paper are: documentary method, interview method, questionnaire method and mathematical statistics method [3].

\section{Research results and analysis}

\subsection{The current situation of the number of school sports injury cases in Jiangxi province}

The article collected 54 cases of school sports injuries in Jiangxi Province from 2013 to 2019. It can be seen from Figure 1 that the number of cases of school sports injuries in Jiangxi province has increased in recent years, of which 16 cases were reported in 2017 (according to statistics, there were only more than 60 cases of school sports injuries in China, and the number of cases in Jiangxi Province reached $20-30 \%$ of the cases of school sports injuries in China) [4], and the number of cases in 2019 the number is up to 15 . In order to avoid the occurrence of school sports injury cases in Jiangxi province, the relevant departments should attach great importance to it, strengthen the management and education, and introduce laws and regulations related to school sports disputes. (Figure 1)

\footnotetext{
* Corresponding author: agaojianxin1985@126.com
} 


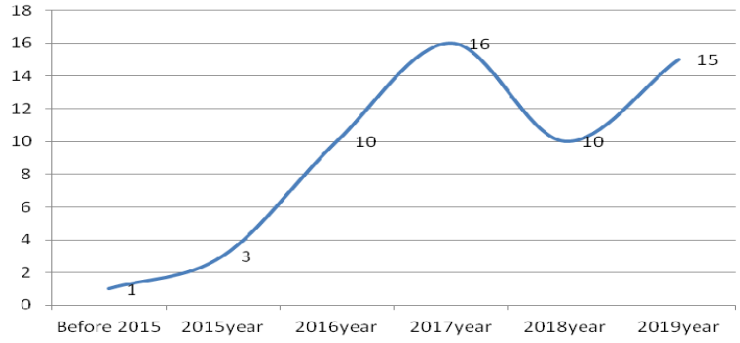

Figure 1. The number and development trend of school sports injury cases in Jiangxi province

\subsection{The current situation of school sports injury cases in Jiangxi province}

From the statistics of 54 cases of school sports injuries in Jiangxi province, we can see that in recent years, 11 cities in Jiangxi province have school sports injuries, including 8 in Jiujiang, 7 in Nanchang, 7 in Ganzhou, 7 in Shangrao, 6 in Fuzhou, 6 in Jingdezhen, 5 in Yingtan, 3 in Ji'an, 2 in Yichun, 2 in Pingxiang and 1 in Xinyu. There are 8 cases of school sports injuries in Jiujiang city, Jiangxi province, and 1 case in Xinyu city. In addition, the data in the figure can be used as a reference for all cities in Jiangxi province, so as to better prevent the occurrence of school sports injury cases [5]. (Figure 2)

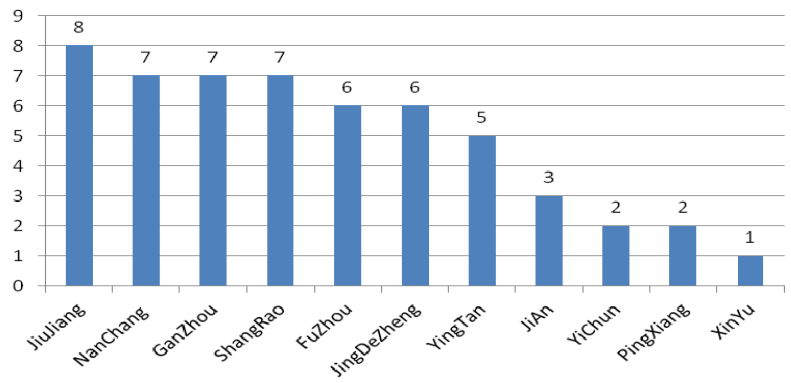

Figure 2. The number of school sports injury cases in Jiangxi province

\subsection{The proportion of sports injury cases in various schools in Jiangxi province}

From the statistics of 54 cases of school sports injuries in Jiangxi Province, it can be seen that in recent years, all kinds of school sports injuries have occurred in Jiangxi province [6]. Among them, there are 8 kindergartens and schools, accounting for $2.4 \%$ of the total number of statistical cases; 22 junior middle schools, accounting for $41 \%$ of the total number of statistical cases; 18 senior middle schools, accounting for $33 \%$ of the total number of statistical cases; 1 University, accounting for $1.2 \%$ of the total number of statistical cases; 2 Private martial arts, dance and other sports schools, accounting for $2.4 \%$ of the total number of statistical cases; 3 Other schools, accounting for $5 \%$ of the total number of statistical cases. Among them, the proportion of school sports injury cases in primary schools and junior middle schools is the largest, which can explain the centralized school area of school sports in Jiangxi province, which is also related to the active and lively characteristics of primary and junior middle school students. All kinds of schools and relevant departments at all levels in Jiangxi province should pay more attention to the school sports safety in this grade. (Figure 3)

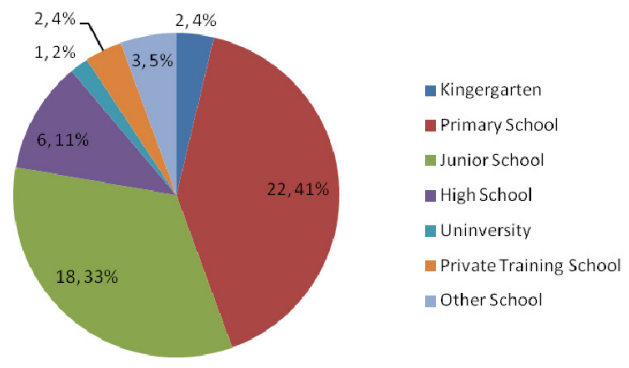

Figure 3. The number and percentage of sports injury cases in various schools in Jiangxi province (unit: /\%)

\subsection{Sports events involved in school sports injury cases in Jiangxi province}

From the statistics of 54 cases of school sports injuries in Jiangxi province, it can be seen that in recent years, school sports injuries in Jiangxi province involve many dangerous sports, including 10 injuries in extracurricular activities, 9 injuries in football, 8 injuries in track and field, 6 injuries in basketball and sports games, 5 injuries in parallel bars and gymnastic equipment, martial arts, taekwondo and others 2 for sports activities, 1 for fishing, badminton, table tennis, tennis, sports dance and indoor physical education. Among them, extracurricular activities, football, basketball, track and field, gymnastics equipment and other sports have the largest number of school sports injury cases. (Figure 4)

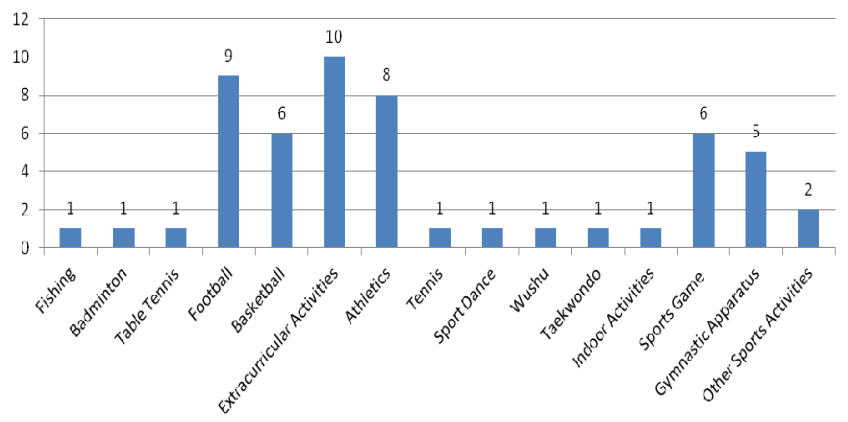

Figure 4. Main sports items involved in school sports injury cases in Jiangxi province

\subsection{The status of disability level of school sports injury cases in Jiangxi province}

It can be seen from the statistics of 54 school sports injury cases in Jiangxi province that in recent years, there are many injury types involved in school sports 
injury cases in Jiangxi province, including 27 fracture cases, accounting for $50 \%$ of the total investigation and statistics cases; 6 eye injury cases, accounting for $11 \%$ of the total investigation and statistics cases; 5 tooth loss cases, accounting for $5.9 \%$ of the total investigation and statistics cases; 5 fall injury cases, accounting for the total investigation and statistics cases $5.9 \%$ of the cases were investigated and counted; 2 cases of contusion,

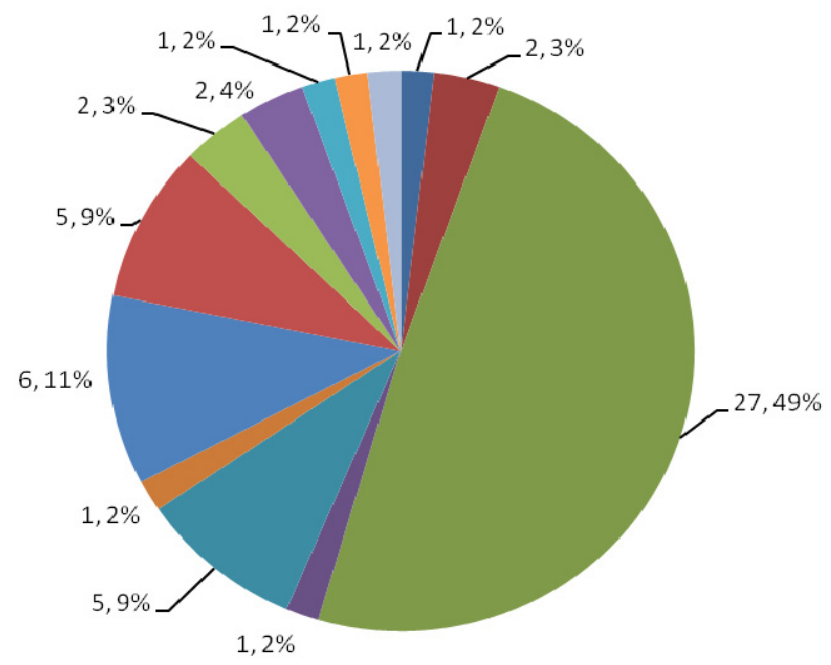

muscle and soft tissue injury and visceral injury, respectively, accounted for $2.3 \%$ of the total investigation and statistics; 1 case of crash, smash, electric injury, disability, organ failure and sudden death, respectively, accounted for $1.2 \%$ of the total investigation and statistics. Among these types of injuries, the number of school sports cases involving fractures is the largest [7]. (Figure 5)

Figure 5. Main types and percentage of disability involved in school sports injury cases in Jiangxi province of students in sports participation; and other factors. The

\subsection{The main reason and responsibility of school sports disputes in Jiangxi Province}

From the statistics to the data of injury cases, we can see that there are many main reasons for the occurrence of school sports injury cases in Jiangxi Province, including: the absence of education and supervision at all levels and all kinds of schools; the main responsibility of physical education teachers; the safety of school sites and equipment; the lack of safety knowledge and awareness

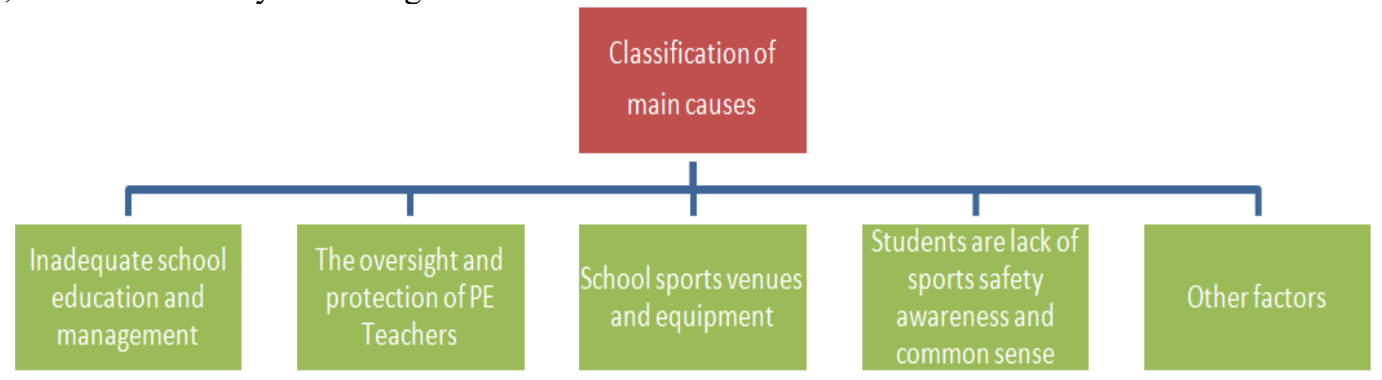

Bruise
Contusion
Eracture
Be injured by a crashing object
- Fall and hurt oneself
Electric injury
Eye injury
Tooth loss
Muscle and soft tissue pull
- Visceral injury
Cripple
Organ failure
Sudden death

Bruise

Fracture

- Be injured by a crashing object

- Fall and hurt oneself

Electric injury

Eye injury injury accidents is the lack of safety awareness of students and the safety knowledge of participating in sports. In these cases, the safety awareness of students is not strong, schools, PE teachers and families lack education for students, students are generally not intentional (non fault liability), but there are also very few provocative students (fault liability) [8]. (Figure 6)

Figure 6. Classification of the main causes of school sports injury cases in Jiangxi Province

\subsection{Statistical analysis on the current situation of school sports injury cases in Jiangxi province}

Through the specific analysis of school sports injury cases in Jiangxi province, the author found that these cases are mostly produced in physical education class, sports meeting and sports competition, extracurricular sports activities and extracurricular training, sports insurance and other cases. Among them, there are up to 24 cases of injuries in all kinds of school physical education classes, accounting for $44 \%$ of the total number of statistical cases; 10 cases of injuries in games and sports competitions, accounting for $18 \%$ of the total number of statistical cases; 11 cases of injuries in extracurricular sports activities and extracurricular training, accounting for $20 \%$ of the total number of 
statistical cases $\%$ There are 7 sports insurance cases, accounting for $13 \%$ of the total number of statistical

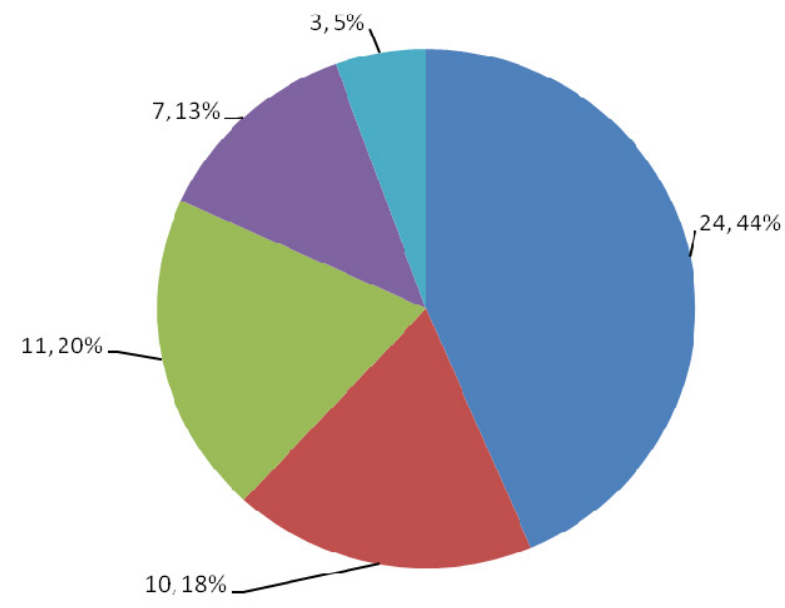

Figure 7. Types and percentage of school sports injury cases in Jiangxi province

\section{Conclusions}

This paper aims to analyze the school physical education injury cases in Jiangxi province and the conclusions are as follows:

\subsection{Pay more attention to the injury cases of school physical education in Jiangxi province.}

It can be found from the cases of school sports injuries in Jiangxi province collected by the civil judgment document network that most of the injured students in these cases have been identified as grade 8 to grade 10 disabilities, with the minor falling, bumping, serious fracture, disability and even sudden death. In addition, in these cases, the amount of compensation and medical treatment is also very high, less than 100000 , more than one million. This has brought a huge physical and mental blow to the victims of school sports cases, brought economic pressure to their families and schools, and seriously affected the students' rights of body, health and life [9]. From the perspective of the number of cases, the 54 cases counted are not many, but these are cases that can be solved by judicial means, which does not mean that these school sports injury cases have occurred in Jiangxi province in recent years, in fact, the number of sports injury events is far greater than the number of statistics.

\subsection{Improve the laws and regulations of school physical education and establish a diversified dispute resolution mechanism.}

Most of the provisions are out of date. The targeted provisions also explain the school sports from the perspective of management and do not legislate from the perspective of students' rights and obligations. In addition, from the perspective of the application of laws cases, and 3 other injury cases, accounting for $5 \%$ of the total number of statistical cases. (Figure 7)

in statistical cases, it can be found that there are almost no rules and regulations on school sports injuries issued by Jiangxi provincial people's congress. At present, only the "Regulations on prevention and treatment of personal injury accidents of school students in Jiangxi province" passed and promulgated by the provincial people's congress in 2015, but the author makes a mathematical analysis of this regulation one by one, none of which is related to school sports injury. Therefore, the local rules and regulations related to school sports injuries need to be improved and revised.

\subsection{To clarify the responsibility of the case, the development of school physical education should not stop eating because of choking.}

In the majority of cases investigated, the responsible person is primary and secondary school students, who are limited in their ability to act. Students can't judge the risk and consequences of sports events. Schools at all levels have the responsibility to carry out education in advance and improve the school sports safety awareness of minors. At the same time, schools have the responsibility to carry out strict school sports management, including venue and equipment, physical education, extracurricular activities, sports competitions and training, etc. On the one hand, strict education management, inspection and injury prevention should be carried out in advance. In a word, due to the large amount of compensation, the school dare not carry out intensive exercise in physical education class, and the organization of sports meeting and sports competition is also small. It only requires nothing to do, or even no physical education class. 


\subsection{It is suggested that the first instance of the local court should solve the case of school sports injury.}

The survey found that in the school sports injury cases counted by the referee network, the number of cases after the second trial is as high as $40 \%$. Most of the cases take a long time to hear and compete, and the cases are concluded only after many court sessions, which brings serious mental and economic burden to students, parents, schools and society. In addition, the result of the second trial is basically to reject the appeal, maintain the original judgment, and only a small part of it has been modified, which also brings great difficulties and difficulties to students' rights protection. In the school sports dispute cases, the plaintiff usually has to appeal to the intermediate people's court to solve the case [10].

\section{Acknowledgment}

This research was financially supported by the humanities and social sciences planning project of universities for Jiangxi province in China (Grant NO. TY19110).

\section{References}

1. Gao Jianxin. A comparative study of sports law between China and the United States [J]. Journal of adult physical education, 2015,31 (06): 21-22 + 48

2. Cheng Jiaquan. A study on the obligation of relief in school sports disputes in the United States [J]. Journal of Chengdu Institute of physical education, 2015,41 (01): 108-113

3. Wang Guoxia. On the mediation mechanism of campus football injury disputes -- from the perspective of the functional limitations of existing dispute resolution methods [J]. Folk law, 2016,18 (02): 267-279

4. Wang Qing. Construction and operation control management of school sports security system [D]. Nanchang Aviation University, 2017

5. Zhu Qirui. Judicial practice and jurisprudential analysis of sports injury cases in Colleges and universities in China [D]. Central China Normal University, 2018

6. Chen Wei. 70 years after the founding of the people's Republic of China: review and Prospect of school sports laws and regulations in China [C]. Chinese Academy of Sports Sciences. Summary of papers of the 11th National Congress of Sports Sciences. Chinese Academy of Sports Sciences: Chinese Academy of Sports Sciences, 2019:30953097Liu Hejun. Applied research in materials and physical engineering construction in Colleges and universities [J]. Bonding, 2019,40 (08): 73-75

7. Zhou Aiguang. The general idea of the revision of China's Sports Law -- an analysis based on the experience of foreign sports legislation $[\mathrm{J}]$. Sports research, 2019,2 (03): 1-7

8. Tian Siyuan. Amendment of sports law and regulations on national fitness from the perspective of "healthy China" [J]. Journal of Shanghai Institute of physical education, 2019,43 (03): 7-12

9. Yang Guoqing, Yan Chengdong. Legal guarantee for the reform and development of China's competitive sports in the new era -- Based on the discussion of the revision ideas and contents of the competitive sports part of the sports law [J]. Sports science, 2019,39 (02): 15-21

10. Gao Jianxin, Fu Baoyan, Wang Yifu, Ma Zhiyong. Research on the current situation and Countermeasures of school sports laws and regulations in China under the background of rule of law [J]. Bulletin of sports science and technology literature, 2019,27 (09): 135-137 